\title{
SATISFAÇÃO COM EXPERIÊNCIAS ACADÊMICAS: VARIÁVEIS PREDITORAS EM ESTUDANTES UNIVERSITÁRIOS DE MOÇAMBIQUE
}

\author{
SATISFACCIÓN COM LAS EXPERIENCIAS ACADÉMICAS: VARIABLES \\ PREDICTORAS EN ESTUDANTES UNIVERSITARIOS DE MOZAMBIQUE
}

\author{
SATISFACTION WITH ACADEMIC EXCPERIENCES: PREDICTOR VARIABLES IN \\ MOZAMBIQUE UNIVERSITY STUDENTS
}

Farissai Pedro CAMPIRA ${ }^{1}$

Paulo Zebo BULAQUE ${ }^{2}$

Leandro Silva ALMEIDA ${ }^{3}$

RESUMO: O presente estudo analisa o impacto das variáveis pessoais e do contexto na (in)satisfação com experiências acadêmicas em estudantes da Universidade Licungo em Moçambique. Participaram do estudo 711 estudantes, sendo 472 (66,4\%) do sexo masculino. Foi aplicado, na modalidade online, um questionário contendo itens de descrição social e acadêmica dos estudantes e de avaliação de satisfação com as experiências acadêmicas. Com base no modelo de regressão hierárquica, a idade dos estudantes, as variáveis de rendimento (e.g., nível de participação nas aulas, intenção de mudança de curso) e as variáveis contextuais (e.g., mudança de residência para frequentar o ES, a ocupação do estudante) mostraram serem preditores significativos da (in)satisfação com experiências acadêmicas. Os resultados do presente estudo sugerem a necessidade de implementação de estratégias de gestão universitária mais focadas nas características individuais e do contexto dos estudantes, visando melhorar a prestação de serviços e a satisfação dos estudantes.

PALAVRAS-CHAVE: Ensino superior. Estudantes universitários. Satisfação acadêmica. Moçambique.

RESUMEN: Este articula analiza el impacto de variables personales y de contexto en la (in)satisfacción con las experiencias académicas por parte de estudiantes de la Universidad Licungo en Mozambique. En el estudio participaron 711 estudiantes, siendo 472 (66,4\%) varones. Se aplicó online un cuestionario que contenía items de descripción social y académica de los estudiantes, así como de evaluación de la satisfacción con las experiencias académicas. Con base en el modelo de regresión jerárquica, la edad de los estudiantes, las variables de desempeño (e.g., nivel de participación en las clases, intención de cambiar de curso) y las variables contextuales (e.g., cambio de residencia para asistir al ES, ocupación del estudiante) se presentarán predictores significativos de la (in)satisfacción de los

\footnotetext{
${ }^{1}$ Universidade Licungo (UL), Beira - Moçambique. Departamento de Educação. Doutorado em Ciências da Educação, especialidade em Psicologia da Educação pela Universidade do Minho. ORCID: https://orcid.org/0000-0002-8131-9907. E-mail: fcampira@live.com

${ }^{2}$ Universidade Licungo (UL), Beira - Moçambique. Departamento de Educação. Doutorado em Psicologia Aplicada pela Universidade do Minho. ORCID: https://orcid.org/0000-0002-6935-6764. E-mail: bulaque@gmail.com

${ }^{3}$ Universidade do Minho (UMINHO), Braga - Portugal. Instituto de Educação. Doutorado em Psicologia, especialidade em Psicologia da Educação pela Universidade do Porto. ORCID: https://orcid.org/0000-00020651-7014. E-mail: leandro@ie.uminho.pt
} 
estudiantes con sus experiencias académicas. Los resultados de este estudio sugieren la necesidad de implementar estrategias de gestión universitaria más centradas en las características individuales y el contexto de los estudiantes, con el objetivo de mejorar la prestación de servicios y la satisfacción de los estudiantes.

PALABRAS CLAVE: Ensenanza superior. Estudiantes universitários. Satisfacción académica. Mozambique.

ABSTRACT: This study analyzes the impact of personal and contextual variables on (in)satisfaction with academic experiences in students from Licungo University in Mozambique. The sample was formed by 711 students, being 472 (66.4\%) male. A questionnaire containing items of students' social and academic description, as well as satisfaction with academic experiences was applied online. Based on hierarchical regression analysis, the students age, the performance variables (eg, level of participation in classes, intention to change course) and contextual variables (eg, leaving home to attend ES, occupation of the student) are significant predictors of (in)satisfaction with academic experiences. These results suggest the need to implement university strategies more focused on individual characteristics and in the academic context to improve the provision of services and students' satisfaction.

KEYWORDS: Higher education. University students. Academic satisfaction. Mozambique.

\section{Introdução}

O ensino superior (ES) recentemente passou a ganhar maior relevância social em Moçambique, principalmente com o aumento da procura por parte dos estudantes e suas famílias, surgindo a necessidade de abertura do ES em instituições privadas (CAMPIRA; BULAQUE; ALMEIDA, 2021). Independentemente da natureza das instituições, os objetivos do ES passam pelo desenvolvimento individual e social dos alunos, assim como pelo desenvolvimento tecnológico e econômico dos países. Para isso, espera-se do ES a criação e disseminação de novos conhecimentos, envolvendo a inovação e a criatividade, assim como a formação científica, técnica, humana e cultural dos seus estudantes (CAMPIRA; BULAQUE; ALMEIDA, 2021; WEERASINGHE; LALITHA; FERNANDO, 2017). Neste sentido, várias reformas e programas de monitoria têm sido desenvolvidos para garantir a qualidade do ES e a qualidade da formação dos seus graduados, ganhando aqui relevância a investigação sobre a satisfação dos estudantes enquanto um dos indicadores da qualidade dos programas, dos processos, das condições e dos serviços das instituições do ensino superior (IES).

O estudo sobre a satisfação não é recente, estando associada à preocupação socioeconômica no sentido de garantir a qualidade nas organizações, oferecendo produtos e serviços que satisfaçam os clientes (BORTOLOTTI et al., 2012; DUTRA; DE MATTOS, 
2020; FORTES et al., 2011; SILVA, 2017; SILVEIRA; DIAS, 2019; WEERASINGHE; LALITHA; FERNANDO, 2017; WEERASINGHE; LALITHA; FERNANDO, 2017).

Este tema no contexto educacional é relativamente recente, sendo que os sistemas de avaliação da qualidade por norma integram a satisfação dos beneficiários (estudantes). Lançando um olhar mais aprofundado, a monitoria da qualidade dos serviços educacionais não é feita apenas em função dos responsáveis institucionais e dos professores, antes considera também a satisfação dos estudantes (RAZINKINA et al., 2018). Embora importante o estudo da satisfação académica no contexto educacional, a centralidade dos estudos tem-se voltado ao Ensino Superior (e.g., ALBUQUERQUE et al., 2019; ALCÂNTARA et al., 2012; ALMEIDA et al., 2020; ASSIS; MOURA; ALVES, 2020), dadas as exigências deste contexto educativo e do mercado de trabalho. Neste sentido, com frequência, os autores nesta área procuram compreender como as expectativas, interesses e experiências dos estudantes são tidos em conta e como a exposição destes às experiências de sua vivência educacional influenciam na sua satisfação, envolvimento e sucesso acadêmico (ALBUQUERQUE; GAMA; BRAGA; VALVERDE; JÚNIOR; COSTA; MILHOMEM; MELO， 2019; ALMEIDA, 2007; ASSIS; MOURA; ALVES, 2020; BRASIL, 2014; LIZOTE et al., 2014).

Centrando-nos no ES, a multiplicidade de IES e a maior diversidade do público estudantil colocam essas instituições em competitividade, assumindo o desafio de responder à multiplicidade de aspirações e interesses dos estudantes. Por outro lado, essa resposta complexifica-se dada a heterogeneidade de estudantes em termos de idades, de origens sociais, de expectativas e de projectos de vida (CAMPIRA; BULAQUE; ALMEIDA, 2021; OSTI et al., 2020). Nesta altura, a forma como as instituições se organizam para dar resposta a essa diversidade, nomeadamente em termos de serviços e clima institucional, é fundamental para a atração, a retenção e o sucesso acadêmico dos estudantes (DUTRA; DE MATTOS, 2020; APPUHAMILAGE; TORII, 2019), assumindo necessariamente a satisfação dos estudantes um dos indicadores da qualidade das IES.

Numa análise dos determinantes da satisfação acadêmica dos estudantes, a investigação sugere a combinação de variáveis pessoais (mais ligadas ao estudante, como por exemplo a sua motivação, interesse, expectativas, idade, sexo ou etnia), de variáveis contextuais (que incluem, por exemplo, a experiência anterior à vida universitária, a experiência vivenciada na universidade, a família, as condições econômicas) e de variáveis institucionais (por exemplo, qualidade das instalações, número e capacitação dos professores, organização dos cursos e serviços), contribuindo todas elas para explicar o sucesso e a satisfação acadêmica (ALBUQUERQUE et al., 2019; ALMEIDA, 2007; CAMPIRA; 
BULAQUE; ALMEIDA, 2021; OSMAN; SAPUTRA; SAHA, 2017; TANDILASHVILI, 2019).

A investigação mostra que a qualidade dos serviços e dos programas formativos impactam na satisfação acadêmica dos estudantes (DUTRA; MATTOS, 2020). Por outro lado, Osti et al. (2020) destacam a qualidade das interações entre professor-estudante, a competência didática dos professores e a percepção da aplicação prática dos conhecimentos aprendidos. Em outro estudo também foi constatado que a interatividade dos docentes, o material de apoio, a temática do curso, as propinas ${ }^{4}$ adequadas e a ligação da universidade com entidades empregadoras influenciam na satisfação dos estudantes (CARVALHO, 2009), surgindo também a atitude dos professores e a capacitação dos mesmos como fatores emergentes da satisfação, sendo os fatores extraclasse menos relevantes (ALCÂNTARA et al., 2012). Mais ainda, Montana (2019) constatou que a imagem corporativa, o valor percebido, a qualidade percebida e a responsabilidade social são determinantes da satisfação dos estudantes.

Uma das linhas de pesquisa relaciona a satisfação acadêmica dos estudantes com o seu desempenho, podendo essa relação ser recíproca (ANTIČEVIĆ et al., 2018; DHAQANE; AFRAH, 2016; KING, 2013; SNOPCE; ALIJA, 2018). A nota de acesso, a escolha do curso, as metas acadêmicas, o autoconceito e a motivação dos alunos, mas igualmente a satisfação acadêmica dos estudantes, encontram-se relacionadas com o desempenho acadêmico, a permanência e a conclusão dos cursos por partes dos discentes (DHAQANE; AFRAH, 2016; FAGUNDES; LUCE; ESPINAR, 2014). Por outro lado, já no contexto moçambicano, o tipo de IES é um fator determinante da satisfação estudantil e do rendimento acadêmico, encontrando-se a satisfação associada ao rendimento acadêmico dos estudantes (NHANTUMBO; CARREÑO; BRUCE-NHANTUMBO, 2018). A satisfação com a formação parece assumir uma função mediadora entre a autoeficácia na formação e o desempenho acadêmico dos estudantes (RIBEIRO, 2019). Mais ainda, em um estudo realizado na Universidade Católica de Moçambique, constatou-se que a qualidade de ensino e a preocupação com a melhoria salarial e desenvolvimento profissional apareciam associados à satisfação acadêmica dos estudantes (MALEQUETA; SANTOS; PERY, 2017).

Assim, com este estudo pretendemos analisar a influência das variáveis pessoais e de contexto na satisfação com experiências acadêmicas em estudantes universitários de Moçambique.

\footnotetext{
${ }^{4}$ No Brasil, mensalidades.
} 


\section{Metodologia}

\section{Participantes}

Participaram neste estudo 711 estudantes, sendo 472 (66,4\%) do gênero masculino e $239(33,6 \%)$ do gênero feminino. No momento de avaliação, as idades dos estudantes variavam de 17 a 59 anos $(M=28.51 ; D P=7.43)$, distribuídos em diferentes faculdades da Universidade Licungo (Faculdade de Educação, 290; Faculdade de Letras e Humanidades, 152; e Faculdade de Ciências e Tecnologias, 269). Quanto às faixas etárias, 294 (41.5\%) tinham idades até 25 anos e 415 estudantes (58.5\%) tinham idade acima dos 25 anos. No que concerne aos locais de frequência do curso de ensino superior, 433 (60.9\%) eram estudantes da Universidade Licungo-Beira e 278 (39.1\%) frequentavam diferentes cursos na Universidade Licungo-Quelimane. Relativamente às ocupações, 344 (48.4\%) referiram ter alguma ocupação além de estudar e o restante são estudantes em tempo integral. Dos participantes, a maioria 412 (57.9\%), tem histórico de reprovação em níveis escolares anteriores (ensino básico e/ou secundário).

\section{Instrumentos}

Foi aplicado, na modalidade online, um questionário contendo questões de descrição social e acadêmica dos estudantes e os itens de avaliação de satisfação com as experiências acadêmicas. O questionário de satisfação é formado por 26 itens distribuídos em quatro dimensões, nomeadamente: a dimensão Curso, Aprendizagem e Rendimento (8 itens; alfa=.87; e.g., o meu nível de participação nas aulas), a dimensão de Infraestruturas e Serviços ( 8 itens; alfa $=.88$; e.g., a qualidade do atendimento pelos funcionários da universidade); a dimensão de Recursos Financeiros (5 itens; alfa=.85; e.g., a suficiência dos meus recursos econômicos para as despesas diárias); e, finalmente, a dimensão de Relacionamento Interpessoal ( 5 itens; alfa=.85; e.g., a quantidade de amigos que tenho no meu curso).

\section{Procedimentos}

O questionário foi aplicado na modalidade online. O preenchimento do referido questionário durou em média 10 minutos. Inicialmente, foram esclarecidos os objetivos do estudo aos estudantes e foi solicitada a participação voluntária destes, assegurando a confidencialidade da informação recolhida e havendo a preocupação em informar que a participação do estudo não afetava os resultados acadêmicos. A coleta dos resultados junto 
dos estudantes durou três semanas. $\mathrm{Na}$ análise dos resultados recorremos ao pacote estatístico SPSS/IBM, versão 20.0, tendo sido efetuada a análise descritiva, a correlação produto $x$ momento de Pearson e o modelo de regressão hierárquica.

\section{Resultados}

Apresentamos a seguir os resultados da presente pesquisa, começando com a análise descritiva das variáveis, em seguida os resultados do estudo correlacional entre a satisfação com as vivências acadêmicas e as variáveis pessoais, contextuais e de rendimento dos estudantes universitários e, por fim, os resultados da regressão hierárquica. Na Tabela 1 apresentam-se os elementos de estatística descritiva para as variáveis em análise.

Tabela 1 - Estatísticas descritivas das variáveis contextuais e de rendimento dos estudantes

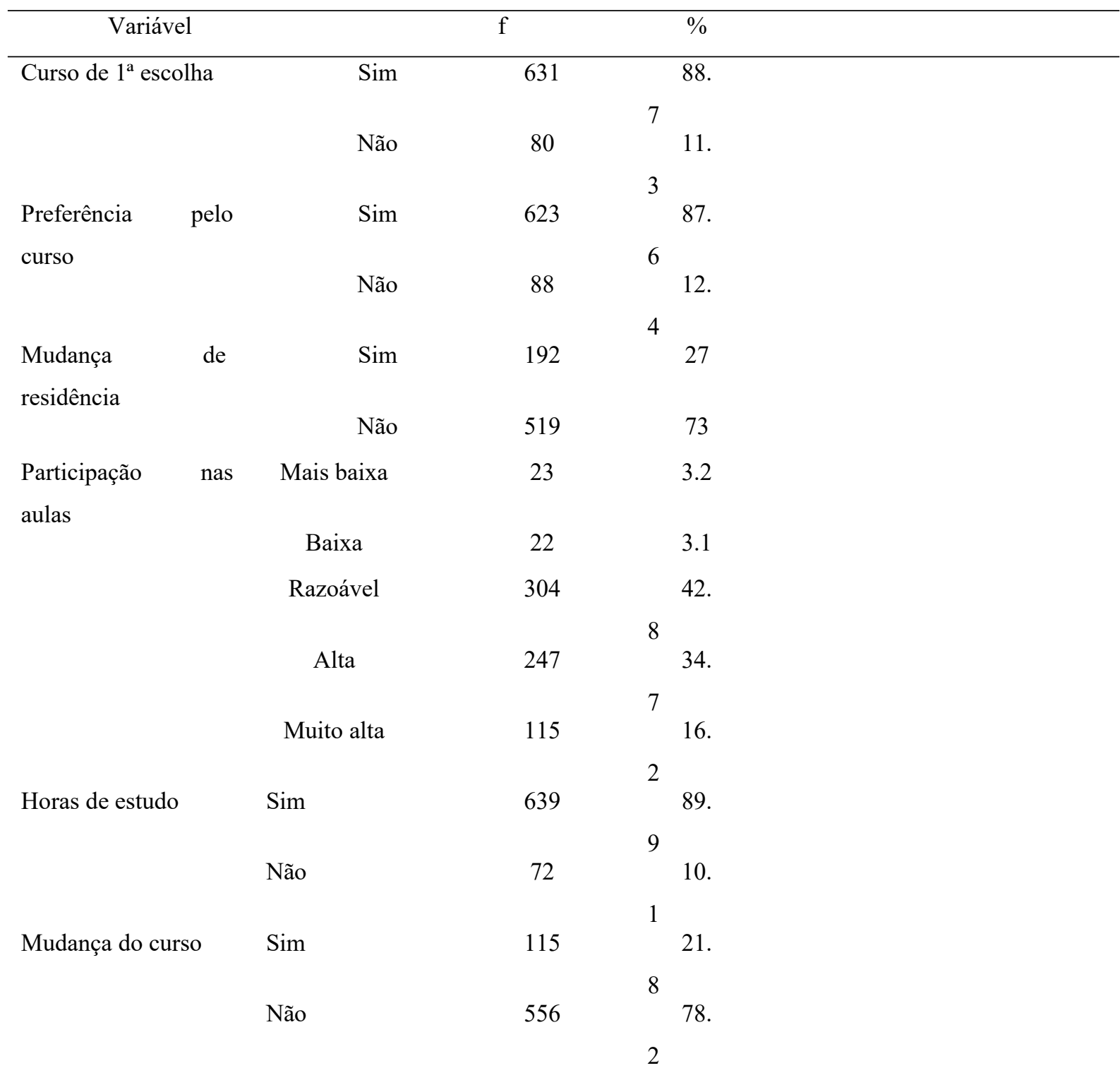


Desistência do curso Sim

149 21

Não

562 79

\begin{tabular}{lcccccc}
\hline & Min & Max & Md & $D P$ & Assimetria & Curtosi \\
\hline Média de acesso no & 2.00 & 17.00 & 10.28 & 2.45 & .25 & .02 \\
ES & & & & & & \\
$\mathrm{N}^{0}$ de faltas as aulas & .00 & 16.00 & .73 & 1.82 & .34 & 24.26 \\
Idade & 17 & 59 & 28.50 & 7.43 & 97 & .93 \\
\hline
\end{tabular}

Fonte: Elaborado pelos autores

Observando os resultados da Tabela 1, a média de acesso dos estudantes no ES variou entre 2 e 17 valores, sendo relativamente fraca $(M=10.28, D P=2.45)$. O número de faltas às aulas variou entre 0 e $17(M=.73, D P=1.82)$. Nesse estudo, a maioria dos estudantes frequentava um curso de $1^{\mathrm{a}}$ opção, 631 (88.7\%) ou o seu curso preferido, $623(87.6 \%)$. Os resultados mostram ainda que poucos estudantes mudaram de residência para frequentar o ES, 192 (27\%). Em termos gerais, a participação dos estudantes nas aulas era razoável, 304 (42.8\%), e a maioria dos estudantes afirmou ter horário individual de estudos, 639 (89.9\%). Adicionalmente, a maioria dos estudantes não pensava mudar de curso, 556 (78.2\%), e nem em desistência, 562 (79\%).

Na Tabela 2 apresentam-se as correlações entre os níveis de satisfação dos estudantes com as suas experiências acadêmicas e variáveis pessoais, contextuais e de rendimento acadêmico.

Tabela 2 - Correlações entre a satisfação com experiências acadêmicas e variáveis pessoais, contextuais e de rendimento dos estudantes

\begin{tabular}{|c|c|c|c|c|c|}
\hline Variáveis & $\begin{array}{c}\text { Curso, } \\
\text { Aprendizagem e } \\
\text { Rendimento }\end{array}$ & $\begin{array}{c}\text { Infraestruturas } \\
\text { e Serviços }\end{array}$ & $\begin{array}{c}\text { Recursos } \\
\text { Financeiro } \\
\text { S }\end{array}$ & Social & $\begin{array}{l}\text { Satisfaçã } \\
\text { o Global }\end{array}$ \\
\hline Idade & $.126^{* *}$ & -.001 & $.091^{*}$ & .056 & $.090^{*}$ \\
\hline Curso de $1^{\mathrm{a}}$ escolha & $-.124^{* *}$ & $-.088^{*}$ & -.006 & -.019 & $-.089^{*}$ \\
\hline Preferência pelo curso & $-.113^{* *}$ & $-.205^{* * *}$ & $-.150^{* * *}$ & $-.148^{* * *}$ & $-.201^{* * *}$ \\
\hline Média de acesso & .074 & .011 & -.013 & .030 & .038 \\
\hline Mudança de residência & $.083^{*}$ & $.146^{* * *}$ & $.249^{* * *}$ & $.102^{* *}$ & $.184^{* * *}$ \\
\hline Histórico de reprovação & -.053 & -.039 & $-.107^{* *}$ & -.064 & $-.082^{*}$ \\
\hline Ocupação do estudante & $-.224^{* * *}$ & $.284^{* * *}$ & $.315^{* * *}$ & $.103^{* *}$ & $.132^{* * *}$ \\
\hline $\mathrm{N}^{\circ}$ de faltas as aulas & $-.133^{* * *}$ & -.055 & .022 & $-.090^{*}$ & $-.089^{*}$ \\
\hline Nível de participação nas & $.181^{* * *}$ & $.125^{* *}$ & $.108^{* *}$ & $.159^{* * * *}$ & $.189^{* * *}$ \\
\hline aulas & & & & & \\
\hline Horário de estudo & .036 & .071 & .020 & .065 & .063 \\
\hline Intenção de mudar do & $.245^{* * *}$ & .058 & -.073 & .072 & $.117^{* *}$ \\
\hline $\begin{array}{l}\text { curso } \\
\text { Intenção de desistir do } \\
\text { curso }\end{array}$ & $.237^{* * *}$ & $.237^{* * *}$ & $.193^{* * *}$ & $.211^{* * *}$ & $.290^{* * *}$ \\
\hline
\end{tabular}




\begin{tabular}{llllll} 
Curso que frequenta & $-.318^{* *}$ & .06 & $.163^{* *}$ & .06 & $-.078^{*}$ \\
\hline
\end{tabular}

Fonte: Elaborado pelos autores

Os resultados da Tabela 2 mostram que, em relação às variáveis pessoais, surge uma associação fraca apenas com a variável idade dos estudantes nas dimensões de curso, aprendizagem e rendimento, na dimensão de recursos financeiros e na medida global de satisfação com experiências acadêmicas, sendo que nestas últimas, a correlação é bastante fraca. Em relação às variáveis de rendimento, a participação nas aulas e a intenção de desistir aparecem mais correlacionadas em todas as dimensões do questionário de satisfação com experiências acadêmicas e a medida global. Mais ainda, o número de faltas às aulas e a intenção de mudar de curso aparecem correlacionados com a dimensão de curso, aprendizagem e rendimento, baixando essa correlação com a dimensão social ou interpessoal e a medida global de satisfação acadêmica. Não foi constatada correlação com significado estatístico tomando a variável horas de estudo dedicados pelo estudante fora das aulas ministradas na faculdade.

Relativamente às variáveis contextuais, surgem correlações estatisticamente significativas nas variáveis preferência do curso, mudança de residência para frequentar o ensino superior e ocupação do estudante, sendo que as correlações nesta última variável são tendencialmente moderadas. Ainda nestas variáveis contextuais surgem também correlações negativas, mas significativas, com a variável de escolha do curso como primeira opção nas dimensões de curso, aprendizagem e rendimento, na dimensão de infraestruturas e serviços universitários e na medida global de satisfação acadêmica. $\mathrm{O}$ histórico de reprovação anterior ao ingresso na universidade apresenta correlação fraca e negativa com a dimensão de recursos financeiros e a medida global. Finalmente, a média de acesso ao ensino superior não se apresenta correlacionada com as dimensões do questionário de satisfação com as experiências acadêmicas.

Num segundo momento do presente estudo, procedemos à análise dos preditores da satisfação acadêmica recorrendo ao modelo de regressão hierárquica. Em cada modelo de predição foram inseridas as variáveis que se mostraram associadas às dimensões da satisfação acadêmica. No primeiro bloco de predição, foi inserida a variável individual idade; no segundo bloco, foram inseridas as variáveis do rendimento, designadamente o número de faltas a aulas na semana passada, o nível de participação nas aulas, a intenção de mudar de curso, bem como a intenção de desistir do curso; e no terceiro bloco, foram incluídas as variáveis contextuais, nomeadamente, o curso de $1^{\text {a }}$ escolha, a preferência pelo curso que 
frequenta, a mudança de residência para frequentar o ES, o tipo ou natureza do curso frequentado e a ocupação do estudante.

Nesta análise, a idade do estudante revelou ser um preditor estatisticamente significativo da satisfação acadêmica, $\beta=.09, p=.017,95 \%[.04, .38]$, explicando $1 \%$ de variância. No segundo modelo, o nível de participação nas aulas e a intenção de mudar do curso mostraram serem preditores estatisticamente significativos da satisfação acadêmica, $\beta=.16, p<.001,95 \%[1.72,4.41] ; \beta=.27, p=<.001,[8.41,14.34]$, explicando $12 \%$ de variância. As restantes variáveis não emergiram como preditores significativos. No terceiro modelo, a preferência pelo curso, a mudança de residência para frequentar o ES, o curso que frequenta e a ocupação do estudante surgiram como fatores preditores significativos da satisfação acadêmica dos estudantes, $\beta=-.14, p=.001,95 \%$ IC $[-11.15,-2.94], \beta=.09$, $p=.017,95 \%$ IC $[.66,6.54] ; \beta=-.09, p=.013,95 \%$ IC $[-.43,-.05] ; e \beta=.11, p=.003,95 \%$ IC [1.38, 6.46], explicando $15 \%$ de variância (Tabela 3 ).

Tabela 3 - Preditores da Satisfação Acadêmica Global

\begin{tabular}{|c|c|c|c|c|c|c|c|}
\hline \multicolumn{2}{|r|}{ Preditores } & $R^{2}\left(R^{2} a j\right)$ & Beta & $t$ & \multirow[t]{2}{*}{1} & \multicolumn{2}{|r|}{$95 \%$ IC } \\
\hline 1 & Idade & & & & & $\cdot$ & {$[.04, .38]$} \\
\hline & & $01(.01)$ & 09 & .40 & 017 & & \\
\hline 2 & & $.12(.12)$ & & & .00 & & \\
\hline & $\mathrm{N}^{\mathrm{o}}$ de faltas as aulas & & .03 & .83 & .407 & & {$[-.95, .39]$} \\
\hline & Nível de participação nas aulas & & 16 & 4.47 & .000 & & {$[1.72,4.41]$} \\
\hline & Intenção de mudar do curso & & 27 & .54 & .000 & & {$[8.41,14.34]$} \\
\hline & Intenção de desistir do curso & & 05 & .39 & .165 & & {$[-.86,5.03]$} \\
\hline 3 & & $.16(.15)$ & & & .000 & & \\
\hline & Curso de $1^{\mathrm{a}}$ escolha & & 05 & .34 & .181 & & {$[-1.34,7.08]$} \\
\hline & Preferência pelo curso & & .14 & 3.37 & .001 & & {$[-11.15,-2.94]$} \\
\hline & Mudança de residência & & 09 & .40 & .017 & & {$[.66,6.54]$} \\
\hline & Curso que frequenta & & .09 & 2.49 & .013 & & {$[-.43,-.05]$} \\
\hline & Ocupação do estudante & & 11 & .03 & .003 & & {$[1.38,6.46]$} \\
\hline
\end{tabular}

Nota: IC=Intervalos de Confiança.

Fonte: Elaborado pelos autores

\section{Discussão}

Os resultados do presente estudo permitiram constatar que as variáveis contextuais, nomeadamente a mudança de residência para frequentar o ensino superior e a ocupação do estudante aparecem correlacionadas com as dimensões de (in)satisfação com as experiências acadêmicas. Procurando atribuir algum significado a esses resultados, a literatura sugere que um dos desafios dos estudantes na transição para o ensino superior está relacionado com a 
mudança de residência, o que implica alguma fragilização dos laços familiares e de amigos de sempre, dificultando ainda mais as suas vivências na universidade, dado o menor suporte socioemocional, principalmente nos momentos iniciais (BENTO; FALCONELLI, 2013; CASANOVA; ARAÚJO; ALMEIDA, 2020; CUNHA; MARQUES; BIAVATTI, 2017; DUARTE; AMARAL, 2019). Por outro lado, com frequência a literatura tem antecipado algumas dificuldades adaptativas e de desempenho dos estudantes que além de estudar tem alguma ocupação, tendo em conta o leque de responsabilidades que tem de gerir, por exemplo, cuidar da família, se dedicar os estudos e atender às exigências do trabalho (DE ALMEIDA; QUINTAS; GONCALVES, 2016; DOUTOR; ALVES, 2019; FERNANDES; OLIVEIRA, 2012; MAIER; MATOS, 2016; VARGAS; COSTA DE PAULA, 2013), associado ao desconhecimento do curso, à dificuldade de realização de atividades escolares e à gestão de problemas familiares e financeiros (DUARTE; AMARAL, 2019).

Os resultados do presente estudo apontaram também que as variáveis de rendimento (participação nas aulas e a intenção de desistir) surgem como as que mais se relacionam com a (in)satisfação com as vivências acadêmicas, sendo que a média de acesso ao ES e as horas dedicadas para o estudo individual não apresentam correlações com significado estatístico. Tomando esses resultados, diferentemente de Matta (2019), que considera os hábitos de estudo, sentimento do curso escolhido e os conhecimentos prévios como condicionantes do desempenho acadêmico, na presente pesquisa, a média de acesso e as horas dedicadas ao estudo não aparecem associadas com os níveis de satisfação dos estudantes com as suas experiências acadêmicas. Por outro lado, há evidências de que existe uma relação direta entre a satisfação acadêmica e o desempenho acadêmico dos estudantes (ALMEIDA et al., 2020; CARMO et al., 2018). Com efeito, a média de acesso não traduz, em si mesma, o desempenho esperado na aprendizagem dos estudantes. Mais ainda, as horas dedicadas ao estudo referenciadas pelos estudantes podem não ser efetivas dada a dificuldade de avaliação dessas aprendizagens em tempo da pandemia, evidenciando dificuldades na gestão de tempo, na gestão de tarefas e na gestão de horários de aulas online (FLORES et al., 2021). Finalmente, nas variáveis pessoais, a preferência por um curso aparece mais associada às dimensões de (in)satisfação com as vivências acadêmicas dos estudantes, sendo também de assinalar a idade que aparece mais correlacionada com a dimensão curso, aprendizagem e rendimento, aproximando-se dos resultados obtidos por Matta (2019).

Tomando agora variáveis mais acadêmicas, surgem em primeiro lugar a escolha do curso, preferência do curso, mudança de residência para frequentar o ES e a ocupação do estudante, em seguida aparecem as variáveis de aprendizagem e de rendimento, 
nomeadamente, o número de faltas às aulas, nível de participação nas aulas, intenção de mudar do curso e intenção de desistir do curso. Estes resultados parecem sugerir que embora a desistência seja um fenômeno global, isso varia de país para país, e de instituição para instituição, sugerindo, desde logo, que as políticas adotadas por cada país ou instituição são essenciais para a retenção dos estudantes, veja-se no Brasil a atribuição de bolsas para favorecer a permanência no ES (BRITO; SOUZA; ALMEIDA, 2021; BRITO; COSTA; AMEIDA; 2019) ou os programa de promoção de resiliência estudantil na Ucrânia (COTTON; NASH; KNEALE, 2017). Por fim, a variável idade é a que menos prediz a (in)satisfação com as vivências acadêmicas dos estudantes, mesmo que em outros estudos as variáveis pessoais (gênero e ser solteiro) e contextuais (baixa renda familiar e ocupação) impactem na evasão do ensino superior (NEGREIROS; COSTA; ALMEIDA, 2021).

\section{Considerações finais}

Os resultados deste estudo permitiram reunir um apartado de informações importantes a ter em conta na Universidade Licungo e que acreditamos serem relevantes na gestão universitária e do processo de ensino-aprendizagem. A primeira indicação é de que estamos diante de grande parte dos estudantes maiores de idade, sugerindo desde logo uma atenção especial na organização das estratégias de ensino-aprendizagem. Mais ainda, as variáveis contextuais como ocupação do estudante, mudança de residência para frequentar o ensino superior, o tipo de curso que o estudante frequenta e a própria escolha do curso aparecem como as variáveis com maior impacto sobre a satisfação com as vivências acadêmicas dos estudantes, seguidas das variáveis de rendimento, nomeadamente, número de faltas e nível de participação nas aulas e intenção de abandonar ou de desistir do curso que frequenta. Tais informações requerem algum cuidado especial, principalmente na organização de serviços de acompanhamento, na disponibilização de informações adequadas de cursos oferecidos, na organização de atividades extracurriculares e na implementação de serviços de apoio aos estudantes na sua adaptação universitária, especialmente aos novos ingressantes.

Em termos de limitações, futuros estudos deverão levar em consideração a análise da satisfação com a experiência acadêmica tendo em conta os cursos frequentados, pois nem todos possuem o mesmo reconhecimento ou prestígio social. Também é importante analisar o fenômeno da (in)satisfação acadêmica considerando os vários anos de frequência dos cursos, podendo antecipar-se mais a satisfação quando unidades curriculares mais práticas ou estágios sejam introduzidos nos anos iniciais do curso. Por último, importa conhecer esta realidade 
acadêmica a partir de estudantes de outras universidades, cobrindo a diversidade de regiões de Moçambique.

\section{REFERÊNCIAS}

ALBUQUERQUE, L. G. M. et al. Satisfação com experiência académica entre estudantes de medicina. Revista Educação em Saúde, v. 7, n. 2, p. 101-110, 2019.

ALCÂNTARA, V. C. et al. Dimensões e determinantes da satisfação de alunos em uma instituição de ensino superior. Revista Brasileira de Marketing, v. 11, n. 3, p. 195-223, 2012. DOI: 10.5585/remark.v11i3.2444

ALMEIDA, L. S. et al. Escala de satisfação no domínio académico em universitários portugueses. Revista Iberoamericana de Diagnóstico y Evaluación, v. 54, n. 1, p. 93-101, 2020. DOI: 10.21865/RIDEP54.1.08

ALMEIDA, L. S. Transicao, adaptação académica e êxito escolar no ensino superior. Revista Galego-Portuguesa de Psicoloxía e Educación, v. 15, n. 2, p. 1138-1663, 2007.

ANTIČEVIĆ, V. et al. Academic achievement and study satisfaction: the contribution of high school sucess and personality. Drustvena Istraživanja. Časopis za opća društvena pitanja, v. 27, n. 2, p. 243-260, 2018. DOI: doi.org/10.5559/di.27.2.03

APPUHAMILAGE, K. S. M.; TORII, H. The impact of loyalty on the student satisfaction in higher education: A structural equation modelling analysis. Higher Education Evaluation and Development, v. 13, n. 2, p. 82-96, 2019. DOI: 10.1108/HEED-01-2019-0003

ASSIS, R. C. C; MOURA, G. L.; ALVES, M. A. Satisfação dos estudantes de Gestão de uma instituição superior pública. ForScience, v. 8, n. 1, 2020. DOI:

10.29069/forscience.2020v8n1.e656

BENTO, A. R.; FALCONELLI, E. F. A dificuldade do jovem na escolha de um curso superior. In: CONGRESSO NACIONAL DA EDUCAÇÃO, 6., 2013, Curitiba. Anais [...]. Curitiba, PR, 2013.

BORTOLOTTI, S. L. V. et al. Avaliação do nível de satisfação de alunos de uma instituição de ensino superior: uma aplicação da teoria de resposta ao item. Gestão \& Produção, v. 19, n. 2, p. 287-302, 2012.

BRITO, A. C. T.; COSTA, A. K. T.; ALMEIRA, V. L. Gestão universitária: políticas comparadas de monitoramento do Programa Bolsa Permanência em universidades federais bresileiras. Revista Ibero-Americana de Estudos em Educação, Araraquara, v. 14, n. esp. 3, p. 1862-1875, 2019. DOI: 10.21723/riaee.v14iesp.3.13036

BRITO, A. C. T.; SOUZA, K. R.; ALMEIDA, V. L. Política pública de assistência estudantil no ensino superior: o programa bolsa permanência na Universidade Federal da Grande Dourados. Revista Ibero-Americana de Estudos em Educação, Araraquara, v. 16, n. 2, p. 508-523, 2021. DOI: 10.21723 /riaee.v16i2.13671 
CAMPIRA, F. P.; BULAQUE, P. Z.; ALMEIDA, L. S. Quem são eles? Características sociodemográficas, de aprendizagem e de rendimento dos estudantes da Universidade Licungo. Revista AMAzônica, v. 8, n. 1, p. 116-133, 2021.

CARMO, T. S. et al. Ensino da disciplina de Contabilidade Geral em cursos de graduação em Administração: Uma análise empírica da relação entre satisfação e desempenho discente.

Revista Contabilidade Vista \& Revista, v. 31, n. 1, p. 105-135, 2020.

CARVALHO, R. J. F. Os fatores determinantes da satisfação dos alunos de mestrados de continuidade. 2009. Dissertação (Mestrado em Marketing) - Instituto Superior de Ciências do Trabalho e da Empresa, Portugal, 2009.

CASANOVA, J. R.; ARAÚJO, A. M.; ALMEIDA, L. S. Dificuldades na adaptação académica dos estudantes do $1^{\circ}$ ano do Ensino Superior. Revista E-Psi, v. 9, n. 1, p. 165-181, 2020 .

COTTON, D. R.; NASH, T.; KNEALE, P. Supporting the retention of non-traditional student in Higher Education using a resilience framework. European Educational Research Journal, v. 16, n. 1, p. 62 -79, 2017. DOI: 10.1177/1474904116652629

CUNHA, L. C.; MARQUES, L.; BIAVATTI, V. T. Adaptação ao ambiente do ensino superior: perceção dos alunos do curso de ciências contábeis. Revista Mineira de Contabilidade, v. 18, n. 1, p. 26-37, 2017.

DE ALMEIDA, A. C. P. F.; QUINTAS, H. L.; GONCALVES, T. I. C. Estudantes nãotradicionais no ensino superior: barreiras à aprendizagem e na inserção professional. Laplage em Revista, Sorocaba, v. 2, n. 1, p. 97-111, 2016.

DHAQANE, M. K.; AFRAH, N. A. Satisfaction of students and academic performance in Benadir University. Journal of Education and Practice, v. 7, n. 24, p. 59-63, 2016.

DOUTOR, C.; ALVES, N. Transição para o Ensino Superior: mudanças e aprendizagens dos estudantes provenientes dos Países Africanos de Língua Oficial Portuguesa (PALOP). In: INTERNATIONAL MEETING OF SOCIOLOGY (ISSOW), 3., 2018, Lisboa. Proceedings [...]. Lisboa, Portugal: Faculdade de Letras, University of Lisbon, 2019. p. 59-71.

DUARTE, R. G.; AMARAL, D. A Licenciatura em Educação do campo da UFES e os desafios da permanência campesina no ensino superior. In: REUNIÃO NACIONAL DA ANPEd, 39., 2019, Niterói. Anais [...]. Niterói, RJ: Universidade Federal Fluminense. 2019. p. 1-7.

DUTRA, V. B. R.; DE MATTOS, V. L. Validade de constructo e confiabilidade: uma escala para a satisfação discente. Boletim online de Educação Matemática, v. 8, n. 15, p. 128-143, 2020. DOI: $10.5965 / 2357724 X 08152020128$

FAGUNDES, C. V.; LUCE, M. B.; ESPINAR, S. R. O desempenho acadêmico como indicador de qualidade da transição Ensino Médio-Educação Superior. Revista Ensaio: Avaliação e Políticas Públicas em Educação, v. 22, n. 84, p. 635-670, 2014. DOI: 10.1590/ S0104-40362014000300004 
FERNANDES, P. D.; OLIVEIRA, K. K. S. Trabalho e Educação: análise reflexiva da dupla jornada do estudante-trabalhador. In: COLÓQUIO INTERNACIONAL EDUCAÇÃO E CONTEMPORANEIDADE, 6., 2012, São Cristóvão. Anais [...]. São Cristóvão, SE: UFS, 2012. p. 1-13.

FLORES, M. A. et al. Ensino e aprendizagem à distância em tempos de COVID-19. Um estudo com alunos do ensino superior. Revista Portuguesa de Pedagogia, v. 55, p. 1-28, 2021. DOI: $10.14195 / 1647-8614551$

FORTES, V. C. et al. Avaliação da qualidade no ensino superior: Aplicação do modelo HEDPERF em uma IES privada. In: CONGRESSO NACIONAL DE EXCELÊNCIA EM GESTÃO, 7., 2011, Rio de Janeiro. Anais [...]. Rio de Janeiro, 2011.

KING, M. D. Motivation and education: performance, commitment, and satisfaction among pharmacy students. Graduate Student Theses, Dissertations, \& Professional Papers, 2013.

LIZOTE, S. A. et al. Satisfação dos acadêmicos com o curso de ciências contábeis: Um estudo em instituições de ensino superior privadas. Advances in Scientific and Applied Accounting, São Paulo, v. 7, n. 3, p. 407-431, 2014.

MAIER, S. R. O.; MATTOS, M. O trabalhar e o estudar no contexto universitário: Uma abordagem com trabalhadores-estudantes. Revista Saúde, Santa Clara, v. 42, n. 1, p. 179185, 2016. DOI: $10.5902 / 2236583420477$

MALEQUETA, A. F.; SANTOS, L. S.; PERY, M. R. M. Análise da Satisfação académica de Estudantes do curso de Educação Física e Desportos do Ensino a Distancia da UCM. Revista Educação a Distância, v. 7, n. 1, p. 73-92, 2017.

MATTA, C. M. B. Influência das vivências académicas e da autoeficácia na adaptação, rendimento e evasão de estudantes nos cursos de engenharia de uma instituição privada. 2019. Dissertação (Mestrado em Psicologia da Saúde) - Universidade Metodista de São Paulo, São Paulo, 2019.

MONTANA, S. Satisfação dos estudantes do ensino superior: O caso da ESTiG do IPBragança vs ESTGA da UAveiro. 2019. Dissertação (Mestrado em Gestão das Organizações) - Instituto Politécnico de Bragança, 2019.

NEGREIROS, J. P.; LAZILLOTTI, R. S; FARIA, C. O. Evasão no ensino superior em cursos presenciais: uma proposta preventiva por meio de um tratamento estatístico. Revista IberoAmericana de Estudos em Educação, Araraquara, v. 16, n. 2, p. 637-648, 2021. DOI: 10.21723/riaee.v16i2.13188

NHANTUMBO, D. J.; CARREÑO, Á. B.; BRUCE-NHANTUMBO, B. S. Satisfação com a educação recebida e rendimento académico em estudantes do ensino superior da cidade da Beira, Moçambique. Revista Científica Electrónica de Edcación y Comunicación en la Sociedad del Conocimiento, v. 18, n. 2, p. 316-334, 2018. 
OSMAN, A. R.; SAPUTRA, R. S.; SAHA, J. Determinants of students satisfaction in the context of higher education: A compete structural equation modeling approach. British Journal of Marketing Studies, v. 5, n. 6, p. 1-14, 2017.

OSTI, A. et al. Satisfação académica de estudantes universitários: Construção de uma escala de avaliação. Educação: Teoria e Prática, v. 30, n. 63, p. 1-13, 2020. DOI: 10.18675/19818106.v30.n.63.s14704

OSTI, A. et al. Satisfação académica: Pesquisa com estudantes brasileiros de uma universidade Pública. Revista E-Psi, v. 9, n. 1, p. 94-106, 2020.

RAZINKINA, E. et al. Student satisfaction as an element of education quality monitoring in innovative higher education institution. E3S Web of Conferences, n. 33, p. 1-8, 2018. DOI: $10.1051 / \mathrm{e} 3$ sconf $/ 20183303043$

RIBEIRO, M. A. F. Fatores preditores do desempenho académico: o caso da motivação, satisfação e autoeficácia. 2019. Dissertação (Mestrado em Gestão de Negócios) - Instituto de Gestão e das organizações de Saúde, Universidade Católica Portuguesa, 2019.

SILVA, J. H. O. Modelos de satisfação de estudantes na educação profissional: Integrando qualidade em serviços, resultados da aprendizagem, empregabilidade, imagem, valor e lealdade. 2017. Dissertação (Mestrado em Gestão de Organizações e Sistemas Públicos) Universidade Federal de São Carlos, São Carlos, 2017.

SILVEIRA, M. B. P.; DIAS, S. Satisfação sobre os serviços da divisão académica do IPS: Um estudo com base no SERVQUAL. 2019. Dissertação (Mestrado em Ciências Empresariais) - Instituto Politécnico de Setúbal, Portugal, 2019.

SNOPCE, H.; ALIJA, S. Student Satisfaction, needs, learning outcome and motivation: a case study approach at a See-university. The Eurasia Proceedings of Educational \& Social Sciences, v. 10, p. 197-202, 2018.

TANDILASHVILI, N. Afctor influencing stuident satisfaction in higher education. The case of a Georgian state university. Research Association for Interdisciplinary, conference proceedings, p. 39-54, 2019. DOI: 10.5281/zenodo.3267486

VARGAS, H. M.; COSTA DA PAULA, M. F. A inclusão do trabalhador - estudante na educação superior: Desafio público a ser enfrentado. Revista da Avaliação da Educação Superior, v. 18, n. 2, p. 459-485, 2013.

WEERASINGHE, I. M. S.; LALITHA, R.; FERNANDO, S. Students' satisfaction in higher education: Literature Review. American Journal of Educational Research, v. 5, n. 5, p. 533-539, 2017. DOI: 10.12691/education-5-5-9

\section{Como referenciar este artigo}


CAMPIRA, F. P.; BULAQUE, P. Z.; ALMEIDA, L. S. Satisfação com experiências acadêmicas: variáveis preditoras em estudantes universitários de Moçambique. Revista Ibero-Americana de Estudos em Educação, Araraquara, v. 16, n. 3, p. 1979-1994, jul./set. 2021. e-ISSN: 1982-5587. DOI: https://doi.org/10.21723/riaee.v16i3.15421

Submetido em: 10/12/2020

Revisões requeridas em: 05/01/2021

Aprovado em: 10/02/2021

Publicado em: 01/07/2021 